\title{
Sexually transmitted diseases in children: HIV infection
}

\author{
Jacqueline Y Q Mok
}

\begin{abstract}
Historical overview
AIDS was recognised in children in 1982, as "unexplained immune deficiency and opportunistic infections". ${ }^{1-3}$ The majority of the infants had mothers who were promiscuous or drug addicted, and who also had AIDS. The importance of transmission of HIV from mother to child was soon established, and until recently, perinatally acquired infections accounted for $80 \%$ of all children in the USA and Europe who were infected with HIV.
\end{abstract}

\section{Epidemiology}

Of 10 million adults throughout the world with HIV infection, $75 \%$ have been infected through heterosexual transmission. This has resulted in an increasing number of women who have been infected, and with it, a rise in the numbers of children. Children under the age of 13 years with AIDS represent about $2 \%$ of all the total cases reported to the World Health Organisation. Reporting cases of AIDS underestimates the size of the problem, and it is estimated that there are about one million children throughout the world infected with HIV.

In sub-Saharan Africa, some 900,000 infants have been estimated by WHO to have been born infected with HIV. In Latin America, about 10,000 children are infected as a result of vertical transmission. The numbers of infected children in South and South-East Asia are not known, but the virus is spreading rapidly through heterosexual intercourse and by the end of this century, more Asians may be infected each year than Africans.

In the USA, the numbers of paediatric AIDS cases rose four-fold between 1985 and 1990 , and at the end of February 1992, there were 3,406 cases of AIDS reported in children, the majority infected as a result of vertical transmission.

In Europe, paediatric AIDS cases are concentrated in Romania, France, Italy and Spain. As of March 1991, 2338 cases had been notified to the WHO Collaborating Center on AIDS, half of whom were Romanian orphans infected by horizontal transmission. ${ }^{4}$ With the exception of Romania, the majority $(77 \%)$ of children have been infected by mother-to-child transmission of HIV.

In the United Kingdom 370 children under 15 years of age have been infected with HIV, $64 \%$ of whom are children with coagulation disorders. ${ }^{5}$ With the introduction of donor screening and heat treatment of blood products, no new cases have been documented where infection occurred through transfusion. Although ónly 107 children were reported to have mother-to-child transmission as a risk factor, the increase in the number of women of child bearing age with HIV infection is likely to result in an increase in the numbers of infected children. Recent surveys of maternal HIV infection, using anonymous unlinked samples from newborn babies, revealed a seroprevalence of 0.59 per 1,000 in the Thames regions and 0.29 per 1,000 in Scotland as a whole. Within these areas, inner London and Edinburgh were shown to have particular problems, with seroprevalence figures of 1.26 and 2.46 per 1,000 respectively, during $1990 .^{67}$

\section{Routes of transmission}

The principle routes of HIV infection, for the paediatric population are: (1) Mother-to-child, or vertical, transmission, (2) Nosocomial spread through the use of inadequately sterilised needles and syringes, (3) Transfusion of unscreened blood and untreated blood products. Child sexual abuse has been associated with HIV transmission in a small number of cases. The importance of this route of transmission has yet to be determined. ${ }^{8}$

Although intrauterine transmission is undisputed, the actual timing of infection is unclear. Descriptions of dysmorphic features suggest early (first or second trimester) infection, ${ }^{9}$ and HIV antigen has been identified in fetal and placental tissue from eighth week abortuses. ${ }^{10}$ On the other hand, reports of children presenting with the slowly progressive form of HIV disease suggest that infection may have been acquired round about the time of delivery.

Several prospective studies undertaken in Europe, USA and Africa of children born to HIV infected women, have reported the rate of mother-to-child transmission of $13-40 \% .^{11-13}$ These variations may be partially explained by different enrolment criteria; different methods used to define HIV infection in the young child; differing lengths of follow-up of the cohort, as well as by differences in the clinical and immunological status of the mothers at the time of delivery. A combination of maternal, fetal and virological factors is likely to influence the risk of HIV transmission from mother to child.

Despite the abundance of infected blood and amniotic fluid during delivery, intrapartum infection has not been documented to occur. Prospective follow-up studies of large cohorts of children born to HIV infected women have not demonstrated a protective effect of Caesarean section. Interestingly, a 
recent report from the International Registry of HIV-exposed twins showed that $50 \%$ of first born twins delivered per vaginum and $38 \%$ of firstborns delivered by Caesarean section were HIV infected, compared with $19 \%$ of second borns delivered by either route. The authors postulated that as the first twin passed through the cervix and birth canal, exposure to maternal blood and fluids resulted in a higher incidence of infection, compared with the second twin. ${ }^{14}$ The relative importance of transmission at delivery is emerging, and deserves further study as there are obvious implications for the interruption of mother-tochild transmission by vaccination.

It has been established that breast feeding during acute HIV infection in the post-partum period poses a risk to the infant. ${ }^{15}$ Although previous prospective studies have failed to recruit sufficient numbers of infants, higher rates of transmission have been reported in breast fed children. A recent analysis of risk factors for mother-to-child transmission of HIV, from the European Collaborative Study, reported on 1,005 children from 19 centres. The rate of vertical transmission was significantly higher in breast fed compared to never breast fed infants $(31 \%$ vs $14 \%$, $\mathrm{p}<0.05) .^{16}$ The balance of evidence appears to favour breast feeding as an important route of transmission of HIV from mother to child, although the exact contribution has not been quantified.

In the United Kingdom, the largest group of children with HIV are boys with haemophilia, infected because of therapy with factor VIII and IX. Since October 1985, universal screening of donors and treatment of blood products have virtually eliminated new cases of transfusion-associated HIV infection. However, in the developing world, transfusion related infection continues to be a problem, owing to the cost of implementing universal screening of donors. Recently, many countries in Eastern Europe have revealed many cases of paediatric AIDS, caused by nosocomial spread by the use of inadequately sterilised syringes and needles. Children have been reported from Romania and the USSR. Horizontal transmission of HIV in large cohorts of young children have not been studied as intensively as children with vertically-acquired disease. It remains to be seen whether the incubation period, natural history and clinical spectrum of horizontally acquired disease in the young child differ from that seen in the child with vertical infection.

\section{Diagnostic methods}

Prospective studies of children born to HIV infected women show that the efficiency of mother-to-child transmission of the virus is about $30 \%$. There is a customary wait of 18 months while maternal IgG antibodies clear, before the child can be classified as being infected or not. Early diagnosis of HIV infection in infants is essential, as therapeutic advances mean that prophylactic treatment against opportunistic infections and antiretroviral therapy can be offered to infected infants. The lack of a single sensitive, specific and practical laboratory test for early diagnosis means that many tests are still being used.

(a) HIV antibody Tests for antibody to HIV using IgG assays remain the most reliable indicator of infection in adults and older children. In most individuals, currently licensed test kits will detect HIV antibody within 4 to 12 weeks of initial infection, although prolonged seroconversion periods have been reported. The transplacental passage of IgG antibodies means that a positive HIV antibody result in a young infant, less than 18 months old, does not necessarily imply infection. In addition, some infected infants are hypogammaglobulinaemic and will not produce antibodies despite being infected. Reports of the detection of HIV specific IgA antibodies in the saliva of infected children may be helpful in early diagnosis. ${ }^{17}$ Another technique, using a modification of antibody testing, detects the presence of HIV-specific antibody-producing $B$ lymphocytes in the infant's circulation. One method for the in vitro antibody production (IVAP) assay is to separate peripheral blood lymphocytes from whole blood, stimulate the lymphocytes and test the culture supernatant with standard methods for antibody detection. ${ }^{18} \mathrm{~A}$ positive antibody test means previous sensitisation of $B$ lymphocytes in an infected infant.

(b) HIV culture The virus can be cultured from peripheral blood mononuclear cells, or plasma. Although a positive culture remains the gold standard diagnostic test, its usefulness is limited as cultures generally take $7-28$ days to complete, are costly and labour intensive, and requires special safety precautions for the laboratory personnel. Also, it requires a relatively large volume of blood, making it less practical in neonatal practice. The decreased sensitivity of the test, due to small volumes of blood or to low virus load in asymptomatic individuals, means that a negative culture result does not necessarily exclude HIV infection.

(c) HIV antigen HIV core antigen can be detected using commercially available kits. In the presence of high titres of HIV specific antibody, however, antigen is complexed to the antibody and is therefore undetectable. Studies of infants born to HIV infected mothers have found very few infants to be antigen positive early in the course of disease, owing to the presence of maternal antibody. ${ }^{19} \mathrm{HIV}$ antigen is usually detected at specific points in the natural history of the disease-prior to seroconversion and in the terminal stages of the disease. The concentration of core antigen correlates with virus load, and is sometimes used to monitor the effect of antiviral therapy; as well as a prognostic marker of disease progression.

(d) Polymerase chain reaction This promising technique amplifies HIV-DNA a millionfold to increase the probability of detection. It allows detection of ten molecules of viral DNA 
in $1 \mu \mathrm{g}$ of human DNA, or one HIV-DNA molecule in 100,000 mononuclear cells.

Although highly sensitive, the method has its limitations, which has prevented its routine use. False positive results arise from contamination, and the technique is labour intensive. Early investigators have reported discordant results when comparing PCR methods with HIV culture and antigen detection. ${ }^{1920}$ It is likely that the sensitivity of PCR varies during the course of HIV infection, being least sensitive when the individual is asymptomatic. $^{21}$

(e) "Surrogate tests" For many children, non-specific laboratory abnormalities usually alert the paediatrician to a diagnosis of HIV infection. Polyclonal hypergamma-globulinaemia has been reported to be an early and specific indicator of HIV infection. ${ }^{1122}$ In the European Collaborative Study about $40 \%$ of infected children showed evidence of raised gammaglobulin levels by three months. By six months, hypergammaglobulinaemia was shown to be a reliable predictor of infection, with a sensitivity $77 \%$ and specificity of $97 \%$. At the same age, low CD4/CD8 ratio was found to have a sensitivity of $31 \%$ and a specificity of $99 \%$. Decreased CD4+ T cells and a low ratio of CD4/CD8 $\mathrm{T}$ cells usually occur late in paediatric HIV disease. However, no large studies have been performed to define the normal ranges for CD4 and CD8 cells in children.

Thrombocytopaenia may be the presenting feature in many HIV infected children. Indeed, these children are often seen by haematologists and treated as "immune thrombocytopaenia" prior to HIV testing.

\section{Clinical presentation}

Children who have haemophilia and HIV infection resemble adults in the incubation period and clinical manifestations of the disease; and their clinical presentation will not be discussed in this section. For those infected by mother-to-child transmission, the mean incubation period is about 2.3 years $(90 \%$ confidence interval 1.9-3.1 years), although children presenting at an older age have been reported. Despite infection occurring in utero, clinical disease is rarely seen in the neonatal period, apart from the dysmorphic features described by workers from New York. ${ }^{9}$ The existence of this "fetal AIDS syndrome" remains controversial.

In perinatally-infected children, retrospective reviews and prospective studies have delineated a bimodal distribution in the clinical spectrum. In the USA, two retrospective reviews ${ }^{2324}$ of cohorts of children showed that $80 \%$ presented with a severe infantile form of the disease. The median age at diagnosis was 8 months; median survival from birth 77 months, and from diagnosis, 38 months. Scott and her co-workers showed that the AIDS indicator disease influenced survival, with Pneumocystis carinii pneumonia claiming the highest mortality in infants less than a year old. ${ }^{23}$ The prospective European Collaborative
Study, confirmed the bimodal clinical picture. ${ }^{11}$ By 12 months, $26 \%$ of 64 infected children had developed AIDS, with a mortality of $17 \%$ during the first year. Thereafter, the disease progression slowed down considerably, with the rest of the infected children remaining stable with the "slowly progressive" form of HIV disease. The natural history of this form of the disease remains to be elucidated, although it is doubtful if natural history studies would remain ethical, in the presence of therapeutic advances. It is more likely that the "unnatural history" will unfold, as antiretroviral therapies are used in children.

For all infected children, the clinical manifestations of the disease are varied and the course variable. Attempts have been made to classify paediatric HIV infection, according to organ involvement, and the one proposed by the Centers for Disease Control ${ }^{25}$ is shown in table 1 .

P2A non specific findings Although common in the early stages of HIV infection, these findings also occur in the absence of HIV infection, especially in children from areas of multiple deprivation and from developing countries. Symptoms include recurrent fever, diarrhoea and failure to thrive, while common signs are lymphadenopathy and hepatosplenomegaly which may resolve spontaneously.

P2B progressive neurological disease HIV involvement of the central nervous system has been reported in up to $90 \%$ of children from the United States, ${ }^{26} 27$ while prospective follow-up of children in the European Collaborative Study found that only $30 \%$ had neurological involvement. ${ }^{28}$ Clinically, affected children present with a loss or delay in acquisition of developmental milestones (over at least 3 months); impaired head growth (either by serial measurement of head circumference, or cerebral atrophy on CT); or progressive symmetrical motor deficits due to abnormal gait, tone or weakness.

The presence of HIV antigen in the CSF in excess of $25 \mathrm{pg} / \mathrm{ml}$ confirms the diagnosis of encephalopathy, but there is poor correlation between clinical severity and laboratory abnormalities in the CSF. It is important to exclude

Table 1 Classification system for HIV infection in children $<13$ years of age

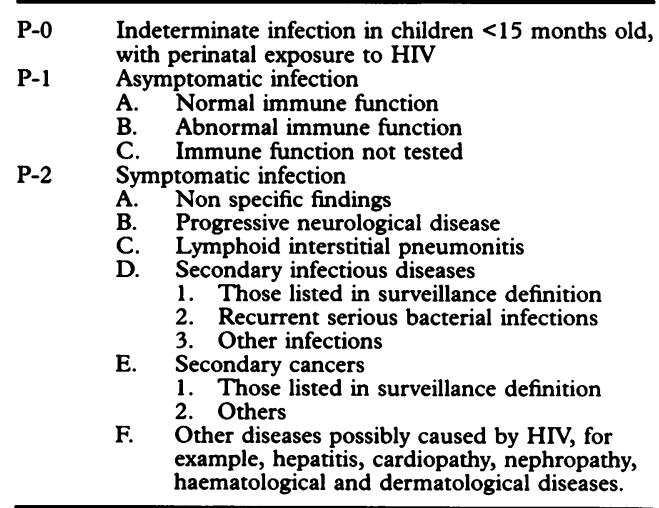


opportunistic infections of the brain as a cause of the neurological signs; however, compared with adults, children rarely have opportunistic infections of the central nervous system.

P2C lymphoid interstitial pneumonitis This debilitating interstitial lung disease is a characteristic feature of AIDS in childhood, occurring in about half of the cases meeting the criteria for AIDS. The onset is insidious, with dyspnoea on exertion and a dry cough. Initially, signs relating to the respiratory system may be minimal, but chronic polyglandular enlargement (including parotids and perihilar nodes) may be present. With disease progression, finger clubbing is seen. The prognosis for children with LIP is better than for those presenting with other AIDS indicator diseases, ${ }^{23}$ although survivors have severely restricted exercise tolerance.

A chest radiograph shows diffuse bilateral reticulonodular interstitial infiltrates with or without perihilar lymphadenopathy. The abnormalities persist despite antimicrobial therapy. A presumptive diagnosis can be made when radiological findings are unchanged or deteriorate over 2 months, and other infective causes (tuberculosis, pneumocystis, CMV) have been excluded. The definitive diagnosis is made by lung biopsy and histological examination, when interstitial infiltrates with lymphocytes and plasma cells are seen.

P2D secondary infectious diseases Despite polyclonal hypergammaglobulinaemia, recurrent serious bacterial infections are common. Children can present with pneumonia, meningitis, abscesses of internal organs, bone or joint infections, and septicaemia. Organisms include streptococcus, pneumococcus, staphylococcus, haemophilus and salmonella species.

Opportunistic infections are the hallmark of AIDS, the commonest being Pneumocystis carinii pneumonia (PCP). The diagnosis of PCP is usually invasive and hazardous in an ill child, but the use of monoclonal antibodies has allowed detection of pneumocystis antigen from nasopharyngeal secretions, obviating the need for bronchoalveolar lavage or lung biopsy in the very sick child. ${ }^{29}$

Oral candida can lead to dissemination throughout the gastrointestinal tract, resulting in anorexia and dysphagia. Other opportunistic organisms which cause disseminated

Table 2 Surveillance programme for infants born to HIV infected women

At each visit,
$\quad$ Review growth, development and signs of
HIV infection
- Discuss child care issues, including
immunisation
- Monitor laboratory results
- Liaise with mother's physician
- Ensure mother understands where and
from whom advice should be sought, if
child is unwell. This is important where
there is shared care.
Referral and liaison if necessary to,
- Paramedical staff
- Education department
- Social work department
- Voluntary agencies/self help groups

disease including cytomegalovirus, herpes simplex virus and atypical mycobacteria.

$P 2 E$ secondary cancers Kaposi's sarcoma, the feature of AIDS in homosexual men, has been reported in $4 \%$ of children. Other cancers which have been described in HIV infected children include lymphoma and Ewing's sarcoma. It is imperative that a national cancer registry is established, so that further malignant disease can be monitored, as the children survive longer. Only then can the long term effects of therapeutic intervention be unravelled.

P2F other diseases Children have presented with conditions such as cardiopathy, nephropathy, thrombocytopaenia, anaemia and dermatitis which have not been definitively attributed to HIV. With time, it is obvious that no organ in the body is spared the effects of HIV, either directly or indirectly. Figure 1 summarises the spectrum of HIV infection.

\section{Follow-up regimes}

In Edinburgh, evidence of HIV infection in pregnant women has concentrated almost exclusively on those who have been intravenous drug users, or whose partners were known to be HIV seropositive due to drug use. ${ }^{30}$ The women live in severely deprived areas of the city, where other existing features predispose to adverse pregnancy outcome and high infant mortality, rather than HIV infection per se. ${ }^{31}$

The unpredictable lifestyles and low level of antenatal care amongst HIV infected pregnant women demand a high level of coordination between medical, obstetric and paediatric staff in order to ensure that both mother and child are followed up. The model in Edinburgh is that of a family clinic, with home visits made if necessary. ${ }^{32}$ The paediatrician is usually introduced to the woman in the antenatal period, to discuss issues surrounding mother-to-child transmission. The paediatrician is present soon after delivery, to examine the child and obtain relevant specimens. Future visits are then arranged.

All infants born to HIV infected women should be followed up intensively in the first year of life, with regular clinical examinations and venesection, in an attempt to make a definitive diagnosis in the infant. Early recognition of HIV infection allows appropriate and aggressive therapy, which may prolong survival. After the age of two years, a proportion of children will be "presumed uninfected", and follow-up can relax to six monthly until the age of 5 years. The ultimate outlook for children born to HIV infected women is unknown, and ideally, all children should be "tagged" in case future illnesses may be HIV related. It is accepted that follow-up should reach adolescent, although in practice, the stigma of the disease usually means that most parents will opt out of follow-up once the children reach school age. Figure 2 outlines one follow-up regime, used in Edinburgh, while table 2 summarises the surveillance programme. 
HIV infection

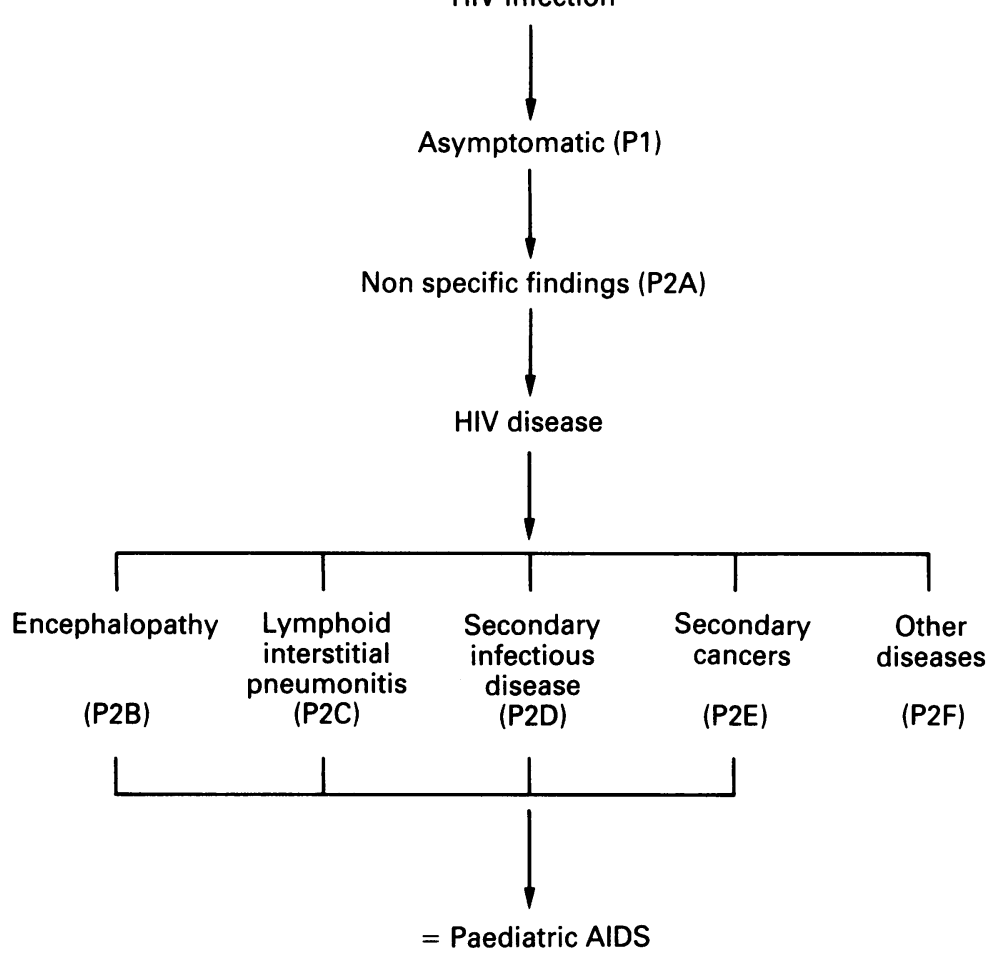

Figure 1 Clinical spectrum of paediatric HIV infection.

\section{Management}

The HIV affected family

The majority of children infected with HIV have acquired the infection from their mother. Consideration must always be given to the health of parents, when child care needs or therapeutic interventions are discussed. With increasing numbers of young parents suffering ill health and requiring frequent hospital admission, close liaison must be developed with colleagues in the Social Work Department so that alternative care can be arranged for the children. The low rate of mother-child transmission in the western world (about 20\%) means that in many families, a proportion of children born to HIV infected women will not be infected, but are by no means unaffected by illness and death in their families. AIDS orphans are those children bereaved by parental death, who may or may not be infected themselves. These children need help to come

Follow-up regime

Infants born to HIV infected women

$$
\text { 1-2 monthly follow-up till }
$$

18-24 months to terms with issues such as drug use, parental life styles, repeated hospitalisations and eventual death in a parent or sibling. Physicians who look after young adults or children with HIV infection need to consider the wider issues of "living with AIDS".

Treatment HIV affects every system, and the underlying principle in therapy is close monitoring of the child so that medically-treatable complications can be identified and treated promptly. Attention must be paid to the growth and nutrition of the child, and expert advice sought from a paediatric dietitian when there is evidence of anorexia, failure to thrive or weight loss.

\section{Prophylaxis}

(a) Recurrent bacterial infections Regular nose and throat swabs allow monitoring of background bacterial flora, so that appropriate antibiotics can be instituted when respiratory infections are diagnosed. The use of regular intravenous immunoglobulin to prevent bacterial infections is used widely, and a recent report from a multicentre double blind placebo trial in the United States revealed the positive benefits of IV IgG therapy. ${ }^{33}$ Children with CD4+ cells of greater than $200 / \mathrm{mm}^{3}$ were found to have benefited most from IV IgG therapy, with a reduction in the number of hospitalisations and an increase in infectionfree intervals.

(b) Pneumocystis carinii pneumonia This is the most common AIDS defining illness in children, and when present in a child less than one year old, has a poor prognosis. In adults, independent predictors of PCP are persistent candidiasis, fever and a CD4 $+\mathrm{T}$ cell count of $<200 / \mathrm{mm}^{3}$. A recent retrospective study in children reported a mortality of $59 \%$, and showed that at the time of diagnosis of PCP, $36 \%$ of the children had CD4 counts of $>$ $450 / \mathrm{mm}^{3}$, with $27 \%$ of infants having counts of $>1,000 / \mathrm{mm}^{3}$. This would limit the predictive value of $\mathrm{CD} 4$ counts. ${ }^{34}$

Recent guidelines for commencing prophylaxis against PCP are shown in Table $3 .^{35}$

Antiretroviral therapy of the antiretroviral agents, most attention has focused on azidothymidine (AZT, zidovudine) which is the only drug with antiretroviral effects licensed to treat HIV infected children at present. The safety, tolerance of and therapeutic response to the

Table 3 Guidelines for initiation of PCP prophylaxis ${ }^{34}$

Primary prophylaxis should be started when the absolute CD4+ count (cells $/ \mathrm{mm}^{3}$ ) is less than that appropriate for the age of the child:

Infected

Presumed uninfected

1-11 months

12-23 month

$2-5$ years
$\geq 5$ years

$\mathrm{CD} 4+\left(\mathrm{cells} / \mathrm{mm}^{3}\right)$

1,500
750
500 $\quad \begin{aligned} & \text { If no absolute count } \\ & \text { available, start PCP } \\ & \text { prophylaxis if }\end{aligned}$

500
200

prophylaxis if
CD $4+<20 \%$

Any child who has had an episode of PCP should be started

6-12 monthly review ? for how long
Review as clinically indicated, at least

3 monthly
Recommended Regime

Trimethoprim $150 \mathrm{mg} / \mathrm{m}^{2}$ and sulphamethoxazole $750 \mathrm{mg} / \mathrm{m}^{2}$ per day, given in two divided doses, three times a week. 
drug were examined in 88 children from nine centres in the United States with advanced HIV disease. ${ }^{36}$ The drug was well tolerated, and although $61 \%$ showed evidence of haematological toxicity, spontaneous resolution occurred although some required dose modification or transfusion. The resultant clinical, immunological and virological improvements were similar to those reported in adults.

Although a paediatric syrup has been licensed for use in many countries, no guidelines exist as to when to start treatment, what dose to give, how frequently to give it and for how long treatment should continue. The dosage has been reported to vary from $360-720 \mathrm{mg} / \mathrm{m}^{2} /$ day, given in $2-4$ doses. A multicentre European trial will commence shortly to assess the effect of early versus deferred use of AZT in children with asymptomatic HIV infection.

Other dideoxynucleosides which have been evaluated only in Phase I and II studies involving children are dideoxycytidine (ddC) and dideoxyinosine (ddI). Agents which compete for attachment to viral protein (gp120) would block viral entry into CD4+ cells, and one such drug under evaluation is recombinant CD4. In theory, each stage of HIV replication is a potential target for therapy, and while many trials are ongoing, the only drug with proven efficacy is zidovudine.

\section{Future developments}

Since reporting of AIDS and HIV infection began in the United Kingdom in 1982, interesting trends have emerged. In the twelve months ending 30.6.91, AIDS cases in men increased by $22 \%$, while a $93 \%$ increase (from 58 to 112 ) was seen in women. ${ }^{5}$ The potential for heterosexual transmission was indicated by the fact that $18 \%$ of AIDS cases and $34 \%$ of HIV infected persons were known to be heterosexual. One way to monitor the spread of the epidemic in the heterosexual population is by serological testing of neonatal dried blood spots. With recent surveys ${ }^{67}$ reporting on the increasing HIV seroprevalence amongst women with live born infants, it is necessary to allocate resources for universal voluntary HIV testing as part of the antenatal programme.

The mechanisms and timing of HIV transmission from mother-to-child require further elucidation. Without knowing the exact time when infection in the fetus occurs, treatment protocols aimed at interrupting transmission cannot be developed. With results of studies suggesting intrapartum infection, ${ }^{14}{ }^{37}$ the possibility exists that passive immunisation or active treatment with antiretroviral drugs given in the newborn period could prevent infection in the neonate.

The advent of new therapies which delay progression of HIV disease makes it imperative that those infected infants born to HIV seropositive mothers be recognised early, so that effective treatment strategies can be offered. At present, the presence of maternal HIV antibodies in the infant's circulation causes delays in diagnosis. Although many diagnostic tech- niques have been used, none have been shown to be useful for practical purposes. There is an urgent need for a comprehensive study to evaluate methods for the early diagnosis of HIV infection within the first six months of life. Also, the predictors of disease progression should be studied in order that those infected children with rapidly progressive disease can be offered treatment. It is likely that future developments in drug treatments will involve combination therapy, where several drugs are used to maximise efficacy while minimising toxicity.

In Africa and many Western countries, the majority of HIV infected children are infected because of mother-to-child transmission. Often, one or both parents are infected and in some families, there may be more than one infected child. The uninfected siblings are affected by illness and death in the family. The HIV affected family, and the millions of AIDS orphans of the future demand a high degree of collaboration between physicians caring for children and adults, hospital and community services, and staff working in social services and education departments. ${ }^{38}$ The resource implications, especially for developing countries, are phenomenal.

1 Oleske J, Minnefor A, Cooper R, Jr, et al. Immune deficiency syndrome in children. $\mathscr{F M} A$ 1983; 249:2345-9.

2 Rubinstein A, Sicklick M, Gupta A, et al. Acquired immunodeficiency with reversed $\mathrm{T} 4 / \mathrm{T} 8$ ratios in infants born to promiscuous and drug-addicted mothers. $\mathscr{f} A M A$ 1983;249:2350-6.

3 Scott GB, Buck BE, Leterman JG, et al. Acquired immunodeficiency syndrome in infants. $N$ Engl $f$ Med 1984; 310:76-81.

4 WHO Collaborating Centre on AIDS. AIDS surveillance in Europe, quarterly report, European Centre for epidemioEurope, quarterly report, European Centre for epidem
logical monitoring of AIDS. Number 29, 31.3.91.

5 AIDS and HIV-1 Infection in the United Kingdom. CDR Report, 24 January 1992;2:4.

6 Ades AE, Parker S, Berry T, et al. Prevalence of maternal HIV-1 infection in Thames Regions: results from anonymous unlinked neonatal testing. Lancet 1991;337: 1562-5

7 Tappin DM, Girdwood RWA, Follett EAC, et al. Prevalence of maternal HIV infection in Scotland based on unlinked anonymous testing of newborn babies. Lancet 1991; 337:1565-9.

8 Gutman LT, St Claire KK, Weedy C, et al. Human immunodeficiency virus transmission by child sexual abuse. Am $\mathcal{f}$ Dis Child 1991;145:137-41.

9 Marion RW, Wiznia AA, Hutcheon G, et al. Human T-cell lymphotropic virus type III (HTLV-III) embryopathy. A new dysmorphic syndrome associated with intrauterine 政

10 Lewis SH, Reynolds Kohler C, Fox HE, et al. HIV-1 in trophoblastic and villous Hofbauer cells, and haematological precursors in eight-week fetuses. Lancet 1990;335:565-8.

11 Children born to women with HIV-1 infection: natural history and risk of transmission. European Collaborative Study. Lancet 1991;337:253-60.

12 Blanche S, Rouzioux C, Moscato ML, et al. A prospective study of infants born to women seropositive for human borns French Collaborative Study Group. $N$ Engl $\mathcal{f}$ Med 189;320:1643-8.

13 Ryder RW, Nsa W, Hassig SE, et al. Perinatal transmission of the human immunodeficiency virus type 1 to infants of 320:1637-42.

14 Goedert JJ, Duliege AM, Amos CI, Felton S, Biggar RJ. The International Registry of HIV exposed twins: a first report. High risk of infection with HIV type 1 for first born, vaginally delivered twins. Lancet 1991;338: $1471-5$

15 Van de Perre P, Simonon A, Msellati P, et al. Postnatal transmission of HIV type 1 from mother to infant: a prospective cohort study in Kigali, Rwanda. NEngl $\mathcal{F}$ Med 1991;325:593-8.

16 The European Collaborative Study. Risk factors for motherto-child transmission of HIV type 1. Lancet 1992; 339:1007-12. 
17 Archibald DW, Johnson JP, Nair P, et al. Detection of salivary immunoglobulin A antibodies to HIV-1 in infants and children. AIDS 1990;4:417-20.

18 Amadori A, De Rossi A, Giaquinto C, et al. In-vitro production of HIV-specific antibody in children at risk of AIDS. Lancet 1988;1:852-4

19 Rogers MF, Ou CY, Rayfield M, et al. Use of the polymerase chain reaction for early detection of the proviral sequenchain reaction for early detection of the proviral sequences of human immunodeficiency virus in infants born to seropositive mothers. New York City Collaborative Study of Maternal HIV Transmission and Montefiore Medical Center HIV Perinatal Tran

20 Laure F, Courgnaud V, Rouzioux C, et al. Detection of HIV 1 DNA in infants and children by means of the polymerase chain reaction. Lancet 1988;ii:538-41.

21 Simmonds P, Balfe P, Peutherer JF, et al. Human immunodeficiency virus infected individuals contain provirus in small numbers of peripheral mononuclear cells and at low copy numbers. $千$ Virol 1990 Feb;64:864-72.

22 Mok JY, Hague RA, Yap PL, et al. Vertical transmission of HIV: a prospective study. Arch Dis Child 1989;64: $1140-5$.

23 Scott GB, Hutto C, Makuch RW, et al. Survival in children with perinatally acquired human immunodeficiency virus type 1 infection. $N$ Engl $₹$ Med 1989;321:1791-6.

24 Krasinski K, Borkowsky W, Holzman RS. Prognosis of human immunodeficiency virus infection in children and adolescents. Pediatr Infect Dis $\mathcal{F}$ 1989;8:216-20.

25 Classification system for human immunodeficiency virus (HIV) infection in children under 13 years of age. MMWR 1987;36:225-30, 235-6.

26 Belman AL, Diamond G, Dickson D, et al. Pediatric acquired immunodeficiency syndrome. Neurologic syndromes. $A m \mathcal{F}$ Dis Child 1988;142:29-35.

27 Epstein LG, Sharer LR, Oleske JM, et al. Neurologic manifestations of human immunodeficiency virus infecmanifestations of human immunodeficiency

28 Neurologic signs in young children with human immuno- deficiency virus infection. The European Collaborative Study. Pediatr Infect Dis $\mathcal{F}$ 1990;9:402-6.

29 Hague RA, Burns SE, Mok JYQ, Yap PL. Diagnosis of Pneumocystis carinii pneumonia from non-invasive sampling of respiratory secretions. Arch Dis Child 1990;65:1364-5.

30 Johnstone FD, McCallum LR, Brettle RP, et al. Testing for HIV in pregnancy: 3 years experience in Edinburgh city. Scott Med $\mathcal{F} 1989 ; 34: 561-3$.

31 Johnstone FD, MacCallum L, Brettle $\mathrm{R}$, et al. Does infection with HIV affect the outcome of pregnancy? $B M \mathcal{F}$ infection with

32 Mok JY, Hague RA, Taylor RF, et al. The management of children born to human immunodeficiency virus seropositive women. $\mathcal{F}$ Infect 1989;18:119-24

33 The National Institute of Child Health and Human Development Intravenous Immunoglobulin Study Group. Intravenous immune globulin for the prevention of bacterial infections in children with symptomatic human immunodeficiency virus infection. $N \mathrm{Engl} f \mathrm{Med}$ 1991;325:73-80.

34 Leiboviz E, Rigaud M, Pollack $\mathrm{H}$, et al. Pneumocystis carinii pneumonia in infants infected with the human carinii pneumonia in infants infected with the human immunodeficiency virus with more than 450 CD4 T lymphocytes per

35 Guidelines for prophylaxis against Pneumocystis carinii pneumonia for children infected with human immunodeficiency virus. $M M W R \quad 1991 ; 40$ RR 2:1-13.

36 McKinney RE, Jr., Maha MA, Connor EM, et al. A multicenter trial of oral zidovudine in children with advanced human immunodeficiency virus disease. The Protocol 043 Study Group. N Engl f Med 1991; 324:1018-25.

37 Ehrnst A, Lingdgren S, Dictor M, Johansson B, et al. HIV in pregnant women and their offspring: evidence for late transmission. Lancet 1991;338:203-7.

38 Mok J. Editorial. HIV infection in children. BMF 1991; 302:921-2. 